\title{
Diels-Alder Cycloaddition of Cyclopentadiene with Ethylacrylate Catalyzed by Mesoporous Al-MCM-48 and Al-MCM-41 Catalysts
}

\author{
Jeong Kuk Shon, ${ }^{\dagger}$ Jae Yi Sim, ${ }^{\dagger}$ Santosh Singh Thakur, ${ }^{\dagger}$ Eun Mi Ko, ${ }^{\dagger}$ Soo Sung Kong, ${ }^{\dagger}$ \\ Ji Yun Choi, ${ }^{,}$Min Kang, ${ }^{*}$ Bidyut Kumar Senapati, ${ }^{\dagger}$ Doo Seoung Choi, ${ }^{\dagger}$ Do Hyun Ryu,${ }^{\dagger,{ }^{*}}$ and Ji Man Kim ${ }^{\dagger+*^{*}}$ \\ ${ }^{\dagger}$ Department of Chemistry and BK21 School of Chemical Materials Science, Sunghowhinan University, \\ Sinwon 440-746, Korea. "E-mail: dhryu@skku.edu \\ ${ }^{\ddagger}$ Functional Materials Laboraton! SKKU Advanced Institute of Nanotechnology, Sunghumkwan University, \\ Suwon 440-746, Korea. "E-mail: jimankim@skku.edu \\ Received July 15,2008
}

\begin{abstract}
In the present work, Diels-Alder reaction of cyclopentadiene with ethylacrylate has been carried out by using two types of mesoporous solid acid catalysts (Al-MCM-41, Al-MCM-48) with different pore structures. The specific topology of Al-MCM-48 (cubic Ia3d structure composed of two independent 3-D channel systems) exhibit higher activity and stereo-control than those of Al-MCM-41 (hexagonal packing of 1-D channels). The physical properties of Al-MCM-48 catalyst, such as high accessibility of reactants to the acid sites, spatial confinement in the nanoscopic reactors, and 3-D channel network stnicture that are effective adsorption and diffusion of reactants, play a crucial tole in the present study.
\end{abstract}

Key Words : Mesoporous catalyst, Al-MCM-48, Diels-Alder reaction, Cyclopentadiene, Ethylacrylate

\section{Introduction}

Diels-Alder (DA) reactions have attracted much attention as a powerful synthesis pathway and widely utilized in synthetic organic chemistry and in the chemical industry, due to the high degree of regio- and stereo-selectivity. ${ }^{1-3}$ It has been reported that the reaction rate, regioselectivity, and stereoselectivity of the DA reactions are markedly affected by homogeneous Lewis acid catalysis. ${ }^{+-6}$ A few solid acid catalysts have been also found to be useful in the DA reaction. ${ }^{7-11}$ In general, the heterogeneous catalysts exhibit several advantages compared with the homogeneous catalysts due to their importance in practical productions of chemicals from the viewpoints of the so-called "zeroemission" or "green" chemistry and ease of separation from the reaction mixture.

The mesoporous materials, designated MCM-41 and MCM-48, are large-porous molecular sieves with pore sizes of about $3 \mathrm{~nm}$, attracting much attention in catalysis and nanoscience. ${ }^{12-14}$ The structure of MCM-48 consists of double gyroidal channels (3-D cubic $103 d$ mesostructure), while the MCM-41 possesses hexagonal packing of straight channels (2-D hexagonal P6mm mesostructure). The 3-D channel network of MCM-48 seems to be much more desirable than the 1-D channels of MCM-41 from the viewpoint of molecular diffusion, adsorption and catalysis. ${ }^{1 \leqslant-17}$ Even though the use of mesoporous aluminosilicate MCM-41 (AlMCM-41) as active catalyst for DA reactions has been reported in the literature, ${ }^{18-20}$ the turnover numbers and selectivities were not encouraging. Surprisingly enough, to the best of our knowledge there is no report on the DA reaction catalyzed with aluminosilicate MCM-48 (Al-MCM48 ), which is probably due to its complicated synthesis manipulations. In continuation of our efforts towards cataly- tic applicability of ordered mesoporous materials, ${ }^{21-2 \varsigma}$ herein we report on the catalytic activity and selectivity over the Al-MCM-48 and Al-MCM-41 catalysts in the DA cycloaddition of cyclopentadiene with ethylacrylate. The AlMCM-48 catalyst exhibits much higher activity as well as stereo-control than those of Al-MCM-41 at low temperature, indicating the advantage of 3-D channel structure.

\section{Experimental Section}

Highly ordered MCM-41 and MCM-48 were prepared according to the methods reported elsewhere. ${ }^{26-29}$ Typically, the pure silica MCM-41 material was obtained by following a synthesis method using cetyltrimethylammonium bromide (CTAB) as structure directing agents. ${ }^{26,27}$ After dissolving $12.15 \mathrm{~g} \mathrm{CTAB}$ in $140 \mathrm{~g}$ of distilled water, the $50 \mathrm{~g}$ of sodium silicate solution $\left(20 \mathrm{wt} \% \mathrm{SiO}_{2}, \mathrm{Na} / \mathrm{Si}=0.5\right)$ was added at room temperature. The mixture was stirred vigorously for 30 min, and then heated to $100{ }^{\circ} \mathrm{C}$ in an oven for $24 \mathrm{~h}$. After cooling the reaction mixture to room temperature, the $\mathrm{pH}$ of reaction mixture was adjusted to 10 using an aqueous solution of $50 \mathrm{wt} \%$ acetic acid. Subsequently, the mixture was heated again to $100^{\circ} \mathrm{C}$ in an oven for $24 \mathrm{~h}$. This cooling$\mathrm{pH}$ adjusting-reheating cycle was carried out twice more. The white powder was filtered, washed in distilled water and dried at $80^{\circ} \mathrm{C}$ overnight. After washing with $\mathrm{HCl} / \mathrm{EtOH}$ mixture of the product and drying at $80^{\circ} \mathrm{C}$, finally calcined in air at $550{ }^{\circ} \mathrm{C}$ for $3 \mathrm{~h}$. The pure silica MCM-48 was prepared using $\mathrm{CTAB}$ and $\mathrm{C}_{12} \mathrm{H}_{25} \mathrm{O}\left(\mathrm{C}_{2} \mathrm{H}_{4} \mathrm{O}\right)_{4} \mathrm{H}$ (Brij 30 , Aldrich), following a synthesis method using cationicnonionic surfactant mixtures. ${ }^{28,24}$ The synthesis procedures of MCM-48 were same with those of MCM-48, except of utilizing the mixture of two surfactants $(10 \mathrm{~g}$ of $\mathrm{CTAB}$ and $1.11 \mathrm{~g}$ of Brij 30 ). 
In order to endow the mesoporous materials with solid acid properties, aluminum was incorporated into the pure silica MCM-41 and MCM-48 materials by grafting method given in the literature ${ }^{30.31}$ Typically, the $1.0 \mathrm{~g}$ of calcined mesoporous powders was stirred in $30 \mathrm{~g}$ of absolute ethanol solution containing $1.33 \mathrm{~g}$ of $\mathrm{AlCl}_{3}$ (Aldrich, 99.999\%) at room temperature for $24 \mathrm{~h}$. The materials were filtered, washed with absolute ethanol, dried at $80^{\circ} \mathrm{C}$ overnight, and finally calcined in air at $700^{\circ} \mathrm{C}$ for $2 \mathrm{~h}$.

Powder X-ray diffaction (XRD) patterns were measured by Rigaku X-ray diffractometer equipped with a $\mathrm{Cu} \mathrm{K} \alpha$ source at $40 \mathrm{kV}$ and $30 \mathrm{~mA}$. Scanning electron microscope (SEM) images were obtained by using Scanning Electron Microscope (Leica Stereoscan 440 ) operating at $15 \mathrm{kV} . \mathrm{X}_{2}$ adsorption-desorption experiments were performed at liquid $\mathrm{N}_{2}$ temperature using a Micromeritics Tristar instrument. Surface areas of the samples were calculated using the standard BET equation, and pore size distributions were estimated by BЛH (Barrett-Joyner-Halenda) method from adsorption branches of isotherms. Elemental analysis for $\mathrm{Al}$ was performed with inductively coupled plasma (ICP) emission spectroscopy (ICPS-1000III)

After being activated under vacuum at $250^{\circ} \mathrm{C}, 100 \mathrm{mg}$ of the calcined Al-MCM-41 or Al-MCM-48 catalysts was introduced in a two-necked round bottomed flask under $\mathrm{N}_{2}$ flow conditions. Immediately, $34.79 \mathrm{mg}$ of ethylacrylate $(0.319 \mathrm{mmol})$ and $2 \mathrm{~mL}$ of $\mathrm{CH}_{2} \mathrm{Cl}_{2}$ were added to the reactor. $65.48 \mathrm{mg}$ of cyclopentadiene $(0.798 \mathrm{mmol})$ was added to the mixture containing the mesoporous acid catalyst after stirring at desired reaction temperature $\left(-35^{\circ} \mathrm{C}-25^{\circ} \mathrm{C}\right)$ for $30 \mathrm{~min}$. The DA reaction was also carried out under the same conditions without any catalyst for comparison. The reaction progress was monitored by Younglin gas chromatography (Autochro-2000 data system) equipped with CycloSil-B column.

\section{Results and Discussion}

XRD patterns for the mesoporous materials, utilized in the

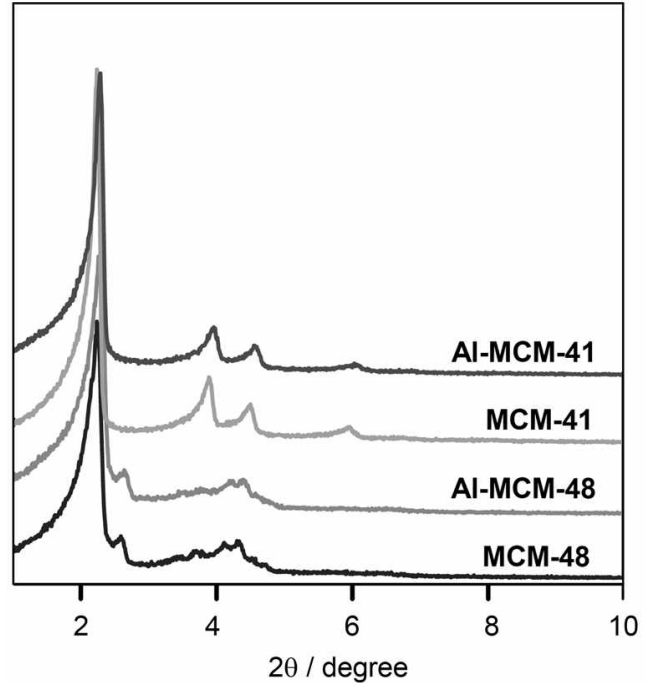

Figure 1. X-ray diffraction pattems of the mesoporous materials utilized in the present work.

present work, are shown in Figure 1. The pure silica MCM41 and MCM-48 materials give several well-resolved diffraction peaks, indicating highly ordered mesostructures of the materials. The MCM-41 shows one intense peak and relatively weak two peaks, which are characteristics of 2-D hexagonal structures $(P 6 m m){ }^{12.13}$ The well-resolved more than five peaks for MCM-48 can be indexed to the bicontinuous cubic la $3 d$ symmetry. ${ }^{12,13,32}$ As shown in Figure 1 , there is no difference in the XRD patterns for both the mesoporous materials after the incorporation of aluminum within the silica framework by the grafting method. $\mathrm{N}_{2}$ adsorption-desorption isotherms and the corresponding B.JH pore size distribution curves also indicate that the present mesoporous materials exhibit well-ordered mesostructures (Figure 2). All the $\mathrm{N}_{2}$ sorption isotherms in Figure 2 are typical type IV isotherms. ${ }^{12-17}$ Well defined steps in the adsorption-desorption curves arrear between the relative pressures, $p / p_{i}$, of $0.3-0.4$, indicating the presence of a narrow distribution of mesopores as shown in the BJH pore
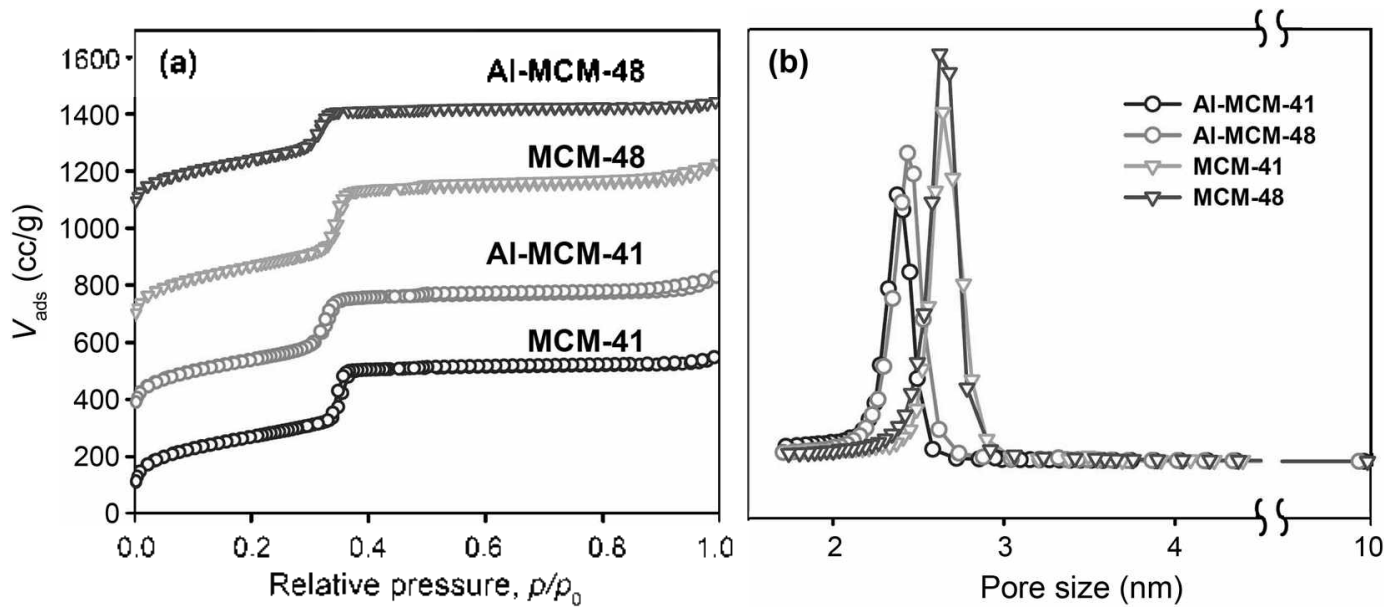

Figure 2. (a) $\mathrm{N}$; adsorption-desorption isotherms of mesoporous materials obtained at liquid $\mathrm{N}=$ temperature, and (b) corresponding BJH pore size distribution curves calculated from adsorption branches. 
Table 1. Physico-chemical properties of Al-MCM-Catalysts

\begin{tabular}{|c|c|c|c|c|c|}
\hline Materials & $\begin{array}{c}\text { Lattice parameter " } \\
\text { (111n) }\end{array}$ & $\begin{array}{c}\text { Surface area } \\
\left(\mathrm{m}^{2} / \mathrm{g}\right)\end{array}$ & $\begin{array}{l}\text { Pore volume } \\
\left(\mathrm{cm}^{2} / \mathrm{g}\right)\end{array}$ & $\begin{array}{c}\text { Pore size }{ }^{i} \\
\text { (nm) }\end{array}$ & $\begin{array}{l}\mathrm{Si} / \mathrm{Al} \text { molar } \\
\text { ratio" }\end{array}$ \\
\hline Al-MCA-4l & 4.45 & 893 & 0.69 & 2.4 & 15.5 \\
\hline Al-MC-M-48 & 9.43 & 881 & 0.82 & 2.4 & 13.1 \\
\hline $\mathrm{MCM}-4 \mathrm{l}$ & 4.55 & 981 & 0.85 & 2.7 & - \\
\hline MCM-48 & 9.65 & 996 & 0.98 & 2.7 & - \\
\hline
\end{tabular}

"Lattice parameters calculated from XRD data using an equation of $a_{0}=d_{100} \times(2 \sqrt{3})$. "Surface areas obtained by the BET method. "Total pore solumes estimated from the $\mathrm{N}$. sorption isothems at $\mathrm{p} / \mathrm{p}_{\mathrm{g}}=0.99$. "Mesopore diameters obtained from the $\mathrm{N}$. adsorption branches using the B.IH method. 'Si/Al molar ratio through ICP analysis.

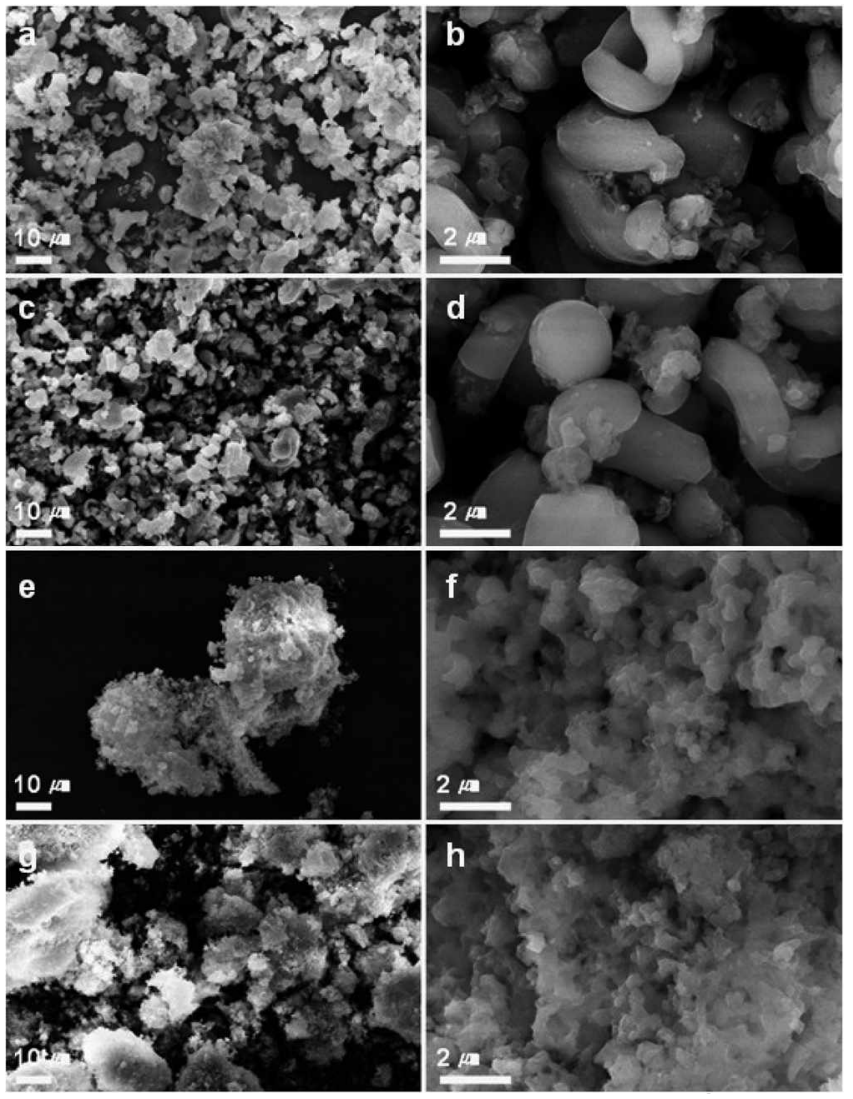

Figure 3. SEM images of mesoporous materials: (a) and (b) MCM$41 ;$ (c) and (d) Al-MCM-41; (e) and (f) MCM-48: (g) and (h) AlMCM-48.

size distribution curves. The physical properties of mesoporous materials are listed in Table 1 . The pore sizes $(2.4 \mathrm{~nm})$ of Al-incorporated mesoporous materials are slightly less than those of the pure silica materials $(2.7 \mathrm{~nm})$, which should be

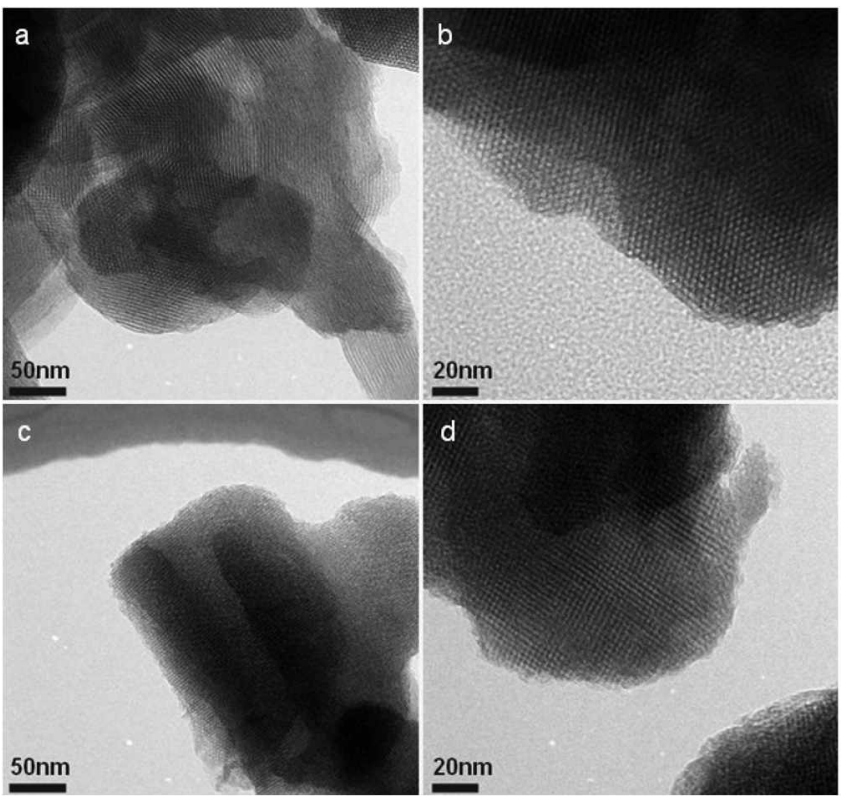

Figure 4. TEM images of mesoporous materials: (a) and (b) AlMCM-41; (c) and (d) Al-MCM-48.

due to the thermal treatment at $700{ }^{\circ} \mathrm{C}$ after the grafting process. The BET surface areas, pore volumes and lattice parameters of the aluminum incorporated materials are also slightly reduced, compared with those of the pure silica materials, as listed in Table 1. Figure 3 shows SEM images of the present mesoporous materials before and after the aluminum incorporation, which indicates that there are no significant changes in the morphologies of the MCM-41 and MCM-48 materials upon the aluminum incorporation. TEM images in Figure 4 clearly show that both Al-MCM-41 and Al-MCM-48 materials exhibit highly ordered mesoporous structures, which coincide with the results of XRD patterns<smiles>C=CC(=O)OCC</smiles><smiles>CCOC(=O)C1CC2C=CC1C2</smiles>

Scheme 1. Schematic diagram for Diels-Alder cycloaddition of cyclopentadiene with ethylacrylate which is catalyzed by mesoporous catalysts containing aluminum within the silica framework. 
and $\mathrm{X}_{2}$ adsorption-desorption isotherms.

As shown in Scheme 1, the Al-MCM-41 and Al-MCM-48 materials were utilized as the acid catalysts for the DA reaction of cyclopentadiene and ethylacrylate. Figure 5 shows the catalytic activities and selectivities for the DA reaction depending on the reaction temperature with and without mesoporous acid catalysts. The DA reaction between cyclopentadiene and ethylacrylate can proceed thermally without requiring a catalyst. However, the rate of the reaction could be increased by introducing a high surface area solid with Lewis acidic sites which may increase the rate of the reaction by concentrating the reactants on the surface. This effect could be enhanced if the solid has nanoscopic pores reactor that can confine the reactants along the reaction coordinate and further decrease the entropy of the activated complex. Very recently, it has been reported that confined nanospaces of micro- and mesoporous materials dramatically affect the stereo-chemical outcome of the reactions. ${ }^{33}$ As shown in Figure 5, the activity of DA reaction at ambient temperature $\left(25^{\circ} \mathrm{C}\right)$ are observed to be $\mathrm{Al}-\mathrm{MCM}-41>\mathrm{Al}-$ MCM-48> blank (without catalyst). At this temperature, the Al-MCM-41 and Al-MCM-48 catalysts show essentially similar catalytic activity and selectivity in the DA reaction. However, very surprisingly, the difference in catalytic activity as well as selectivity of Al-MCM-48 and Al-MCM-41, is dramatic in the DA reaction of cyclopentadiene and ethylacrylate at lower temperature and in the order of Al-MCM$48>\mathrm{Al}-\mathrm{MCM}-41>$ blank (no reaction). Lowering the reaction temperature decreases dramatically the catalytic activity of Al-MCM-41, where as the Al-MCM-48 catalyst give a relatively high catalytic activity above $60 \%$ even at $-35^{\circ} \mathrm{C}$. Based on the catalytic activity in Figure 5, it can be presumed that the interconnected 3-D channel system of $\mathrm{Al}$ MCM-48 is more favorable for the catalytic reaction than the 2-D hexagonal channels of Al-MCM-41, from the viewpoint of diffusion and adsorption/desorption of reactants within the confined nanochannels.
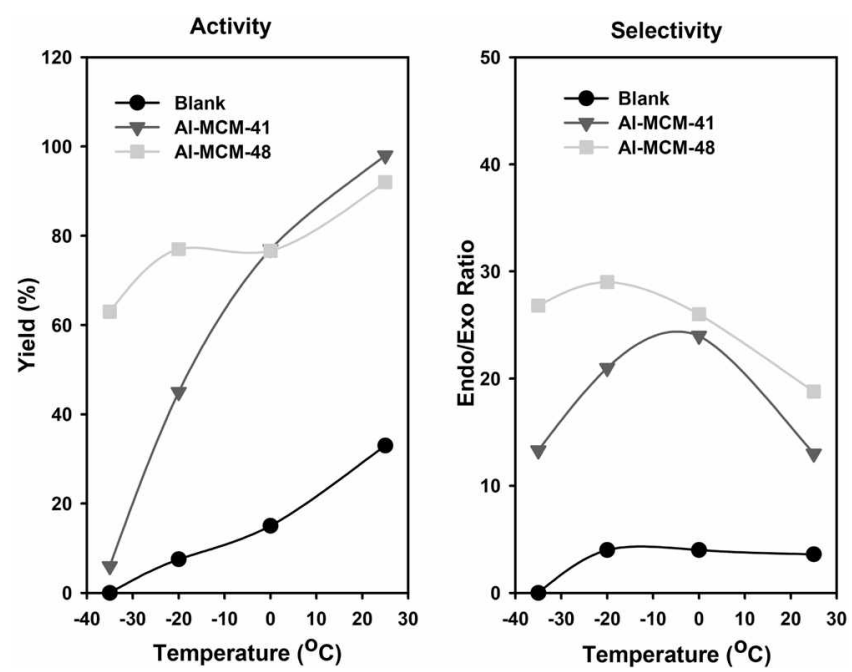

Figure 5. Catalytic activity and product selectivity of the mesoporous catalysts for the Diels-Alder reaction of cyclopentadiene with ethylacrylate at various temperatures.
Figure 5 also shows the product selectivity (endo-lexoisomer ratio) in the DA reaction of cyclopentadiene with ethylacrylate depending on the reaction temperatures. It is well known that DA reaction between simple reactants lead preferentially to the endo product, especially when the diene is cyclic due to the existence of a stabilizing overlap (secondary orbital interaction) between the frontier molecular orbitals of the reactants at centers that do not participate in the formation of $\sigma$ bonds in the endo approach ${ }^{34,35}$ As shown in Figure 5, the DA reaction without catalyst results in the endo-product selectivity (endo-exo-isomer ratio of about 4 ), which may be determined thermodynamically. The DA reaction is an orbital-controlled pericyclic reaction, which is generally carried out with an electron-rich 1,3diene and an electron-deficient dienophile. Lewis acids are known to lower the LCMO of dienophiles, that should increase the selectivity for the endo-product. ${ }^{+20}$ In the present work, both the Al-MCM-41 and Al-MCM-48 catalysts result in the dramatic increase of endo-product selectivity for the DA reaction, compared to that of the reaction without catalyst (Figure 5). The maximum endo-/exo-isomer ratios are obtained to about 29 at $-20{ }^{\circ} \mathrm{C}$ and about 24 at $0{ }^{\circ} \mathrm{C}$ for Al-MCM-48 and Al-MCM-41 catalysts, respectively. Similar to the case of catalytic activity, the Al-MCM-48 catalyst gives no significant decrease in the product selectivity (endo-/ exo-isomer ratio of about 27) upon lowering the reaction temperature to $-35^{\circ} \mathrm{C}$, where as the selectivity falls down to about 13 in the case of Al-MCM-41. This is probable due to the differences in the catalytic activity of the two mesoporous catalysts at low temperature.

\section{Conclusions}

The mesoporous materials containing aluminum within the silica frameworks exhibit an excellent catalytic activity as well as product selectivity for the Diels-Alder raction of cyclopentadiene with ethylacrylate. The Al-MCM-48 catalyst results in the much higher catalytic activity and endoproduct selectivity at low reaction temperature than those of Al-MCM-41 catalyst, which is probably due the 3-D channel structures of Al-MCM-48 from the diffusional point of view. The Al-MCM-48, exhibiting the well-developed regular 3-D mesopores, high surface areas and solid acid property, are expected to give great potentials for various kinds of catalytic applications.

Acknowledgments. This work was supported by the Korea Research Foundation Grant funded by the Korean Government (MOEHRD, KRF-2005-005-J11901).

\section{References}

1. Corey, E. I. Angen: Chen. In. Ed. 2002, 41, 1650.

2. Takao, K.; Munakata, R.; Tadano, K. Chem. Rev: 2005, 105, 4779.

3. Griffiths, G J.; Previdoli, F. E. J. Org. Chem. 1993, 58, 6129.

4. Houk, K. N.; Strozier, R. W. J. Am. Chem. Soc. 1973, 95, 4094.

5. Futatsugi, K.; Yamamoto, H. Angen: Chem. Int. Ed. 2005, 44. 1484. 
6. Fringuelli, F.; Girotti, R.; Pizzo, F.; Vaccaro, L. Org. Leff. 2006, 8 , 2487.

7. Laszlo, P. Acc. Chem. Res. 1986, 19, 121.

8. Fraile, J. M.; García, J. l.; Mayoral, J. A.; Tamai, T.; Alonso, P. J. Chem. Commun. 1996, 1981.

9. Onaka, M.; Yamasaki, R. Chem. Lett. 1998, 259.

10. Chiba, K.; Hiorano, T; Kitano, Y.; Taba, M. Chem. Commun. 1999,691 .

11. Gerstberger, G; Palm, C.; Anwander, R. Chem. Euf, J. 1999, 5 , 997.

12. Kresge, C. T.; Leonowicz, M. E.; Roth, W. J.; Vartuli, J. C.; Beck; J. S. Notute 1992, 359, 710 .

13. Beck, I. S.; Vartuli, I. C.; Roth, W. I.; Leonowicz, M. E.; Kresge, C. T.; Schmitt, K. D.; Chu, C. T.-W.; Olsen, D. H.; Sheppard, E. W; McCullen, S. B; Higgins, J. B.; Schlenker, J. L. J. An. Chem. Soc. 1992, $114,10834$.

14. Coma, A.; Hennenegildo, G. Adv. Sinth Catal. 2006, 348, 1391.

15. Monnier, A.; Schuth, F.; Huo, Q.; Kumar, D.; Margolese, D.; Maxwell, R. S.; Stucky, G. D.; Krishnamurty, M.; Petroff, P; Firouzi, A.; Janicke, M.; Chmelka, B. F. Science 1993, 26l, 1299.

16. Ko, C. H.: Ryoo, R. Chen. Commun. 1996, 2467.

17. Kim, J. M.; Kim, S. K.; Ryoo, R. Chem. Commen. 1998, 259

18. Kugita, T.; Jana, S. K.; Owada, T.; Hashimoto, N.; Onaka, M.; Namba, S. Appl. Catal. A 2003, $245,353$.

19. Satsuma, A.; Segawa, Y; Yoshida, H.; Hattori, T. Appl. Cotcl. A 2004, 264,229

20. Gomez, M. V.; Cantin, A.; Corma, A.; de la Hoz, A. J. Mol. Cartol.
$A 2005,240,16$.

21. Burri, D. R.; Jun, K.-W.; Kim, Y.-H.; Kim, J. M.; Park, S.-E.; Yoo, J. S. Chem. Lett. 2002, 212.

22. Joo, S. H.; Pak, C.; You, D. J.; Lee, S.-A; Lee, H. I.; Kim, J. M.; Chang, H.; Seung, D. Electrochimica Acta 2006, 52, 1618.

23. Park, S. Y.; Choi, B. H.; Kang, M.; Kim, J. M.; Lee, I.-M. J. Mol. Cotal. A 2007, 265, 323 .

24. Jeon, J.-K.; Lee, H.; Yim, J.-H.; Kim, Y. S.; Lee, S. J.; Park, Y.-K.; Shon, J. K.; Kim, J. M. Catal. Lett. 2007, 19, 179.

25. Lee, D.; Kim, H.; Kang, M.; Kim, J. M.; Lee, I.-M. Bull. Korean Chem. Soc. 2007, 28, 2034.

26. Kim, J. M.; Kwak, J. H.; Jun, S.; Ryoo, R. J. Phls. Chem. 1995, 99,16472

27. Ryoo, R.; Kim, J. M. J. Chent. Soc. Chem. Commm. 1995, 711.

28. Ryoo, R.; Joo, S. H.; Kim, J. M. J. Phls. Chent. B 1999, 103, 7435 .

29. Kim, J. M.; Ryoo, R. Bull. Korean Chem. Soc. 1996, 17, 66 .

30. Ryoo, R.; Jun, S.; Kim, J. M.; Kim, M. J. Chem. Commm 1997. 2225 .

31. Ryoo, R.; Jun, S. J. Catol. 2000, 195, 237.

32. Kin, J.; Choi, M.; Ryoo, R. Bill. Korem Chem. Soc. 2008, 29, 413.

33. Song, C. E.; Kim, D. H.; Choi, D. S. Eur. J. Inorg. Chem. 2006, 15,2927 .

34. Sauer, I.; Sustmann, R. Angen: Chen. Int. Ed. 1980, 19,779.

35. Wannere, C. S.; Paul, A.; Herges, R.; Houk, K. N.; Schaefer III, H. F.; Schleyer, P. V. R. J. Comput. Chem. 2007, $28,344$. 